\title{
PARTICIPACIÓN SOCIAL EN LA PROTECCIÓN ACTIVA DE LOS ESPACIOS AGRARIOS PERIURBANOS: UN ESTADO DE LA CUESTIÓN ${ }^{1}$
}

\author{
Alberto Matarán Ruiz \\ Departamento de Urbanística y Ordenación del Territorio. Universidad de Granada. \\ mataran@ugr.es
}

\section{RESUMEN}

La deteriorada situación de las agriculturas y, en particular, de los espacios agrarios periurbanos, implica la necesidad de un cambio del rumbo impuesto por el modelo metropolitano contemporáneo a partir de las oportunidades que brinda la participación social entendida como el gobierno del territorio por parte de la ciudadanía. Para demostrar el interés y las posibilidades existentes, se realiza una descripción sistemática de experiencias que identifican una tendencia que están suponiendo la conservación y dinamización de los espacios agrarios periurbanos resistiendo a las dinámicas territoriales actuales.

Palabras clave: agricultura periurbana, participación social, planificación, conciencia de lugar.

\footnotetext{
ABSTRACT

Agriculture and in particular periurban agriculture are threatened. However, in this article it is proposed a switch in the direction imposed by the contemporary metropolitan model based on the opportunities given by social participation (ruling the territory by citizenship). In order to demonstrate the interest and existing possibilities, it is presented a systematic

Fecha de recepción: diciembre 2011.

Fecha de aceptación: septiembre 2012.

1 Este artículo es resultado del Proyecto de Investigación «Integración del Paisaje en los Procesos de Planificación: Aplicación al caso Andaluz P09-5369», financiado por la Junta de Andalucía en su convocatoria de Proyectos de Excelencia cuyo investigador principal es Alberto Matarán Ruiz, y del Proyecto de Investigación «El Patrimonio Agrario: la construcción del territorio a través de la actividad agraria HAR2010-15809» del Plan Nacional de I+D+i, cuyo investigador principal es Jose Castillo Ruiz.
} 
description of space experiences that are promoting the periurban agrarian spaces and thus resisting to the actual territorial tendencies.

Key words: periurban agriculture, social participation, planning, place awareness.

\section{INTRODUCCIÓN: ARGUMENTARIO CONTRA LA DEGRADACIÓN DE LOS ESPACIOS AGRA- RIOS PERIURBANOS}

Los espacios agrarios periurbanos son, como su propio nombre indica, lugares cercanos a las zonas urbanizadas (Roda Noya, 2009) donde se agudizan por tanto los conflictos que tradicionalmente afectan al medio agrario rural. La voracidad del modelo metropolitano contemporáneo (Magnaghi, 2011) genera grandes tensiones sobre unos lugares que hasta hace pocos años constituían una parte esencial de las ciudades, ya que son una pieza clave para la sostenibilidad de los sistemas de poblamiento. El crecimiento urbano (residencial, comercial e industrial), la incesante construcción de infraestructuras, y la degradación de los lugares de contacto entre los espacios urbanos e infraestructurales y su entorno (United Nations Environmental Program, 2005; European Environment Agency, 2006; Davis, 2006; Fernández Durán, 2006), implican un crecimiento paralelo de la condición periurbana de los espacios agrarios al mismo tiempo que chocan frontalmente con el derecho a existir de los espacios agrarios periurbanos preexistentes y de las gentes que los han creado, los han cuidado y los quieren seguir cuidando en el futuro.

Aparte de las amenazas del modelo metropolitano contemporáneo, se plantean tres dudas fundamentales para abordar los procesos de degradación de los espacios agrarios periurbanos: la supuesta marginalidad de las agriculturas periurbanas, la supuesta carencia de instrumentos de gestión y planificación, y la supuesta carencia de experiencias de gestión y planificación exitosas y duraderas. El principal objetivo de este artículo es tratar de responder a estas tres cuestiones, y para ello se adelanta en los párrafos siguientes un argumentario básico que va a guiar las tesis planteadas.

\subsection{Primera: Sobre la supuesta marginalidad de las agriculturas periurbanas}

La supuesta (e inducida) marginalidad de las agriculturas, de sectores del empresariado agrícola y del campesinado (Van der Ploeg, 2010) ha sido utilizada para situar esta parte esencial de nuestra memoria biocultural (Toledo y Barreda-Basols, 2008) en clara desventaja con respecto a otros usos propios de la metrópolis contemporánea, donde el carácter no empresarial de gran parte de las agriculturas periurbanas (o por tamaño de parcela o por necesidades alimentarias) implica una mayor desconsideración si cabe de un uso del suelo desvinculado de la especulación financiera que domina la economía mundial (Naredo, 2009; Observatorio Metropolitano, 2011).

Sin embargo, desde hace años se están generando experiencias que contradicen la marginación de las agriculturas y de las personas que a ellas se dedican, sobre todo en el espacio periurbano, debido a varias cuestiones como: el desplazamiento de las agriculturas industriales a zonas alejadas, la fragmentación de la propiedad, y las necesidades/deman- 
das de las propias ciudades (Jarosz, 2008). Así, en los países periféricos los llamados ajustes estructurales propios de la imposición de políticas neoliberales han dado lugar a una degradación sin precedentes de las ciudades (Davis, 2006) y a un enorme desarrollo de la capacidad de supervivencia cuya base han sido las agriculturas urbanas y periurbanas (Latouche, 2007) con las que está relacionado un 70\% de la población urbana de África y un $60 \%$ de la de Asia (Bryld, 2003) y que, según la FAO (2007), en 2000 producían entre el 15 y el $20 \%$ de la alimentación mundial; mientras tanto en los países centrales se está dando un número creciente de procesos que se podrían inscribir dentro de lo que en el mundo anglosajón se ha denominado Agriculura Cívica (Civic Agriculture) (DeLind, 2002) o Iniciativas Agroalimentarias Alternativas (Alternative Agrifood Initiatives) (Allen, FitzSimmons, Goodman, y Waner, 2003; Jarosz, 2008; Sánchez Hernández, 2009) destacando la relocalización a través de la utilización de canales cortos de comercialización y la consideración de las múltiples funciones que cumplen las agriculturas como bien común (Renting, Marsden, y Banks, 2003; Donadieu, 2008), poniéndolas en valor en tanto que patrimonio ambiental y cultural.

En este artículo se va a hacer una descripción de estas experiencias que están vinculadas a tres cuestiones centrales para la consideración no marginal de los espacios agrarios periurbanos: la primera es la posibilidad de generar empleo ajeno a las vicisitudes de los flujos de capital y cerca de donde se encuentra la mayor cantidad de población en paro, cuestión defendida en numerosos estudios que demuestran el carácter estratégico del empleo vinculado a la recampesinización para la sostenibilidad de nuestros territorios (Smit, Ratta, y Nasr, 1996; Altieri y Nichols, 2000; Toledo y Barreda-Basols, 2008; González de Molina, 2009; Van der Ploeg, 2010); la segunda, que también tiene que ver con la autonomía, será la cuestión de la soberanía alimentaria, incluyendo tanto la cantidad como la calidad de los productos que consumimos (Bryld, 2003, FAO, 2007; Sánchez Hernández, 2009; Vivas, 2010); y la tercera, será la perspectiva de la calidad de vida, en relación con el disfrute de entornos saludables y con el uso de las agriculturas como un elemento de regeneración urbana y de transformación social (Rubino, 2008; Luginbühl, 2008; Forns Puch, 2008), algo cada vez más extendido en el campo de las relaciones sociales, de la inserción social, de la educación, de la salud...

\subsection{Segunda: sobre la supuesta carencia de instrumentos de gestión y planificación}

La falta de instrumentos y la dificultad para su aplicación en la gestión y la planificación constituye la segunda cuestión clave para una correcta aproximación a la protección dinámica de los espacios agrarios periurbanos.

Sin embargo, existe un número creciente de investigaciones aplicadas en el campo de la planificación, de las ciencias ambientales, de la agronomía, de la ecología, del paisaje, del patrimonio, de la sociología, de la antropología... que plantean instrumentos innovadores que están al servicio de quien quiera utilizarlos, tal y como ya ocurre de hecho en numerosas experiencias, algunas de las cuales se van a describir en este artículo (DeLind, 2002; Valenzuela, Pérez Campaña, Aguilera Benavente, y Matarán, 2007; Donadieu, 2008; Dewaelheyns y Gulinck, 2008; Valenzuela Montes, Pérez Campaña, y Matarán Ruiz, 2009; Fanfani, 2009). 


\subsection{Tercera: sobre la supuesta carencia de experiencias exitosas y duraderas}

La planificación urbana, territorial y sectorial, en tanto que referencia espacial de las políticas públicas (y de poderosos intereses privados), se ha hecho eco de la tendencia a marginalizar las agriculturas y ha sido colaboradora necesaria de los desmanes producidos tanto en nuestras ciudades (Fernández Durán, 2006; Fariña Tojo, 2011) como en el caso particular de los espacios agrarios periurbanos, llegando hasta el punto de que una amplia mayoría de las agriculturas urbanas (y también por tanto las periurbanas) en los países periféricos se siguen considerando ilegales (Bryld, 2003). Todo esto supone un gran problema para el desarrollo de las agriculturas periurbanas que tienen además su mayor limitación en el acceso al suelo, tanto por su escasez como por su consiguiente carestía en los contextos urbanos marcados por la especulación urbanística.

A pesar de esta situación generalizada, no es cierto que no haya suficiente experiencia para abordar adecuadamente la complejidad de los espacios agrarios periurbanos mediante su planificación y su gestión en un contexto complejo y altamente tensionado por los intereses especulativos. Muchos documentos de planificación reconocen los valores de estos espacios con figuras de protección, y en ocasiones se han desarrollado medidas para hacer efectivas estas figuras planteando una aproximación dinámica y potenciando las agriculturas periurbanas sobre todo en los países centrales y en algunos países periféricos como Cuba, Argentina, Zambia o Uganda (Altieri, Companioni, Cañizares, Murphy, Rosset, Bourque y Nicholls, 1999; Bryld, 2003; Benencia, Quaranta, y Souza Casadinho, 2009). Tal y como se va a describir en este artículo, el éxito duradero de algunas de estas políticas públicas en lugares y condiciones diversas, contradice este tercer argumento.

La conclusión fundamental que se puede obtener de los párrafos anteriores es que la degradación actual de la mayoría de los espacios agrarios periurbanos se amplifica como resultado de la típica conjunción triple entre los intereses de personas y grupos poderosos, la voluntad de nuestros gobernantes, y la connivencia o la cobarde indolencia de los cuerpos técnicos de la administración y las empresas, de tal manera que entre todos estos actores se actúa en contra del interés público (Naredo, 2008) o de lo que globalmente se llama el 99\%, en algunos casos sin ni siquiera plantear las excusas que se han descrito. Lógicamente, también hay excepciones muy valiosas, tal y como se va a tratar en este artículo.

Si quienes gobiernan y gestionan el territorio no persiguen el interés público si no los intereses del poder aunque hayamos respondido a (casi) todas sus dudas-excusas, cabe esperar que la implicación de la ciudadanía (o por lo menos de los sectores con conciencia de lugar) permita vislumbrar un horizonte diferente. Esta será otra de las cuestiones claves a abordar en este artículo, ya que es algo que atraviesa desde hace tiempo las discusiones que se dan en el ámbito de la planificación y la gestión territorial, tanto en el campo académico-científico (Dewaelheyns y Gulinck, 2008; Baldeschi y Poli, 2008; Cruz Gallach, 2008; Fanfani, 2009; Michelini, 2010), como en el campo de las experiencias institucionales y sociales que se describirán más adelante.

\section{LA PARTICIPACIÓN SOCIAL COMO OPORTUNIDAD PARA LOS ESPACIOS AGRARIOS PERIURBANOS}

Ante la agudización de los problemas ambientales (como la propia degradación de los espacios agrarios periurbanos) es esencial desarrollar nuevas vías para la promoción de la sos- 
tenibilidad (Riechmann, 2009), que deberán ser locales (Latouche, 2009; Serreau, 2010) aunque hace tiempo que sabemos que se deben relacionar en red (Riechmann y Fernández Buey, 1994; Magnaghi, 2011) para conseguir transformaciones globales (Garnier, 2006; Vivas, 2011). Ahora bien, tratando de fomentar otras maneras de hacer que eviten los errores cometidos, se propone en este artículo la consideración de la participación social como uno de los elementos innovadores que permitan superar la situación actual (Michelini, 2010), entendiendo la idea de la participación como la implicación democrática de la ciudadanía en los asuntos que atañen al gobierno (en un sentido amplio) de sus lugares mucho más allá del mero ejercicio del voto.

Siguiendo las tres conclusiones que describe José Carmona Gallego (2004) con respecto a la exitosa experiencia participativa del Parque Miraflores en Sevilla, se ha generado un argumentarlo que apoya esta idea de la participación social como oportunidad: «1) Cambiar la ciudad (y se podría decir también el territorio) desde los intereses ciudadanos es posible»; «2) La participación ciudadana es un instrumento para la reapropiación de la ciudad (y del territorio)» ; y «3) Protagonizar colectivamente la transformación del territorio requiere ir más allá de la reivindicación de los cambios, asumiendo espacios de soberanía y autogestión».

Atendiendo al postulado número 1, se puede defender que la participación social es esencial para ejercer de contrapeso con respecto al poder establecido y de este modo evitar ciertas actuaciones públicas y/o privadas que afectan a un territorio mediante la presión a las personas que lo gobiernan o lo transforman (Nel·lo, 2006; Cruz Gallach, 2008).

Según los postulados 1 y 2, también hay otros ejemplos en los que la participación social da lugar a propuestas en positivo de tal manera que la implicación de la ciudadanía consigue que se diseñen y se activen determinadas políticas públicas en un territorio, aunque esto sea más difícil que conseguir evitar un plan o un proyecto ideado por una administración y/o por una entidad privada.

En el caso de que las personas que gobiernan un territorio se decidan a dar el paso de aplicar una política pública más o menos «contradictoria» con lo establecido, que ponga en cuestión el poder o que implique ciertas innovaciones con respecto a la dinámica general descrita en el apartado anterior, aparecen múltiples problemas que ponen en peligro el éxito del proyecto. La lógica y la experiencia (ver los ejemplos de este artículo) indican que en estas condiciones una participación social podría suponer una importante reducción de estos problemas, ya que considerando los tres postulados en su conjunto si se consigue la implicación de las personas que viven y cuidan de un lugar, será más fácil abordar con éxito una gestión y planificación de dicho territorio orientada hacia la sostenibilidad. Y esto es todavía más evidente en el caso de los espacios agrarios, ya que son lugares que necesariamente tienen que ser construidos y cuidados por las personas, a diferencia de otros lugares como las propias ciudades en las que la capacidad de transformación y la propia intervención de la ciudadanía está mucho más limitada.

Además, si se produce la circunstancia (frecuente) de que el problema de la aplicación de una política pública radique en un cambio en las personas que gobiernan un territorio, en una involución de la voluntad de las mismas y/o en un cambio de la coyuntura socioeconómica, la participación social podría ser una garantía para que esta política pública se active o para que se siga desarrollando adecuadamente a pesar de los cambios. Es más, si se atiende al tercer postulado, puede darse el caso de que las políticas públicas sean irrelevantes para el mantenimiento de las agriculturas periurbanas, ya que éstas serían cuidadas de forma soberana y autogestionaria por parte de las personas que operan en dicho territorio. El ejemplo 
del Parque Agrario del Sur de Milán que se describe más adelante será paradigmático con respecto a esta capacidad de resistencia ligada a la participación social.

Aparte de estos argumentos, cabe destacar que la participación social que constituye una reivindicación histórica de los movimientos sociales en general y del ecologista en particular (Riechmann y Fernández Buey, 1994; Martínez Alier, 1995), está cada vez más presente en la vida de nuestros territorios, y se apoya en gran medida en el creciente predominio de la conciencia de lugar (DeLind, 2002; Desmarais, 2008; Cruz Gallach, 2008; Magnaghi, 2011). Este proceso de toma de conciencia y de incremento de la participación, está siendo refrendado tanto en numerosos estudios y proyectos (algunos de los cuales serán referenciados a lo largo de este texto), como en documentos institucionales entre los que destaca el Convenio Europeo del Paisaje que es paradigmático respecto a la participación en el ámbito del viejo continente, y que además considera como paisajes de gran importancia a los espacios periurbanos debido entre otras cuestiones a su carácter cotidiano. Además, en el caso particular de la agricultura periurbana en este ámbito, existe un Dictamen del Comité Económico y Social Europeo (2004) que otorga un especial interés a las cuestiones ligadas a la participación, tanto de las personas dedicadas a la agricultura como de otros grupos interesados.

Sin embargo, hasta el momento y con algunas excepciones, las instituciones han orientado la participación social mediante procesos de consulta sobre planes y proyectos previamente definidos (Garnier, 2006; Cruz Gallach, 2008; Pérez Serrano, Castaño Madroñal, y Manjavacas Ruiz, 2009; Magnaghi, 2011) y atendiendo principalmente a lo que Magnaghi (2011) denomina como agentes fuertes del territorio, es decir, aquellos que tienen acceso a la política, a la información, a los recursos económicos y culturales, y a las redes de comunicación.

Es necesario por tanto alterar la ponderación tradicional que se hace de las diferentes opiniones, no sólo para democratizar la democracia, y empezar a considerar a la ciudadanía como personas maduras y capaces (Biehl y Bookchin, 2009), si no para reclamar también una atención especial a quienes tienen algún interés porque los espacios agrarios periurbanos sigan existiendo.

Lógicamente, la participación de la que se está hablando no se trata de la clásica búsqueda del consenso (consensus building), si no de ir mucho más allá. No se trata por tanto de consensuar algo ya decidido si no de desarrollar en el territorio planes y proyectos que sean compartidos (y también construidos) por la mayoría de la sociedad. Y para ello es necesario contar con una diversidad de agentes que, en igualdad de condiciones entre agentes fuertes y débiles, puedan construir escenarios de futuro para el lugar que habitan. No todas las experiencias presentadas en este artículo atienden a todos estos argumentos; sin embargo, la suma de los ejemplos que se describen a continuación, y sobre todo algunos de sus éxitos, aportan una perspectiva de un futuro que podría desarrollarse siguiendo algunas de las ideas que aquí se defienden.

\section{EXPERIENCIAS DE PARTICIPACIÓN SOCIAL EN ESPACIOS AGRARIOS PERIURBANOS}

Para aproximarse al papel de la participación social en los espacios agrarios periurbanos, se han descrito algunas de las experiencias que ya existen en la materia (tanto españolas como internacionales) destacando las características que han permitido la protección y dinamización de los espacios agrarios periurbanos a través de la participación social, de este modo se puede confirmar la existencia de tendencias que contradicen las dinámicas 
aparentemente dominantes de la globalización y el modelo metropolitano contemporáneo. El método utilizado ha sido la revisión bibliográfica y documental, que ha aportado mayor rigor al conocimiento previo de la mayoría de los casos por parte de quien escribe este texto.

Así mismo, para facilitar su comprensión las experiencias se han sistematizado siguiendo tres tipologías cuya descripción se va a realizar al abordar cada una de éstas: Experiencias ciudadanas; experiencias de pactos entre la administración y la ciudadanía; y experiencias de planificación.

Por experiencias ciudadanas, se entienden las experiencias de reivindicación e intervención directa y normalmente (bastante) autónoma por parte de la ciudadanía en los espacios agrarios periurbanos. Esto no significa que sean experiencias ajenas a la administración, pues, excepto en algunos casos de mayor autonomía, mantienen relaciones (normalmente duales) de colaboración y confrontación con los poderes públicos. De ahí que el análisis comience por esta categoría, pues las dos categorías siguientes incluirán en numerosas ocasiones la participación social a través del papel fundador y dinamizador de estas experiencias ciudadanas.

Con respecto a las experiencias de pactos entre la administración y la ciudadanía, se trata de casos en los que los poderes públicos, los agentes económicos y los grupos ciudadanos se ponen de acuerdo para trabajar por un territorio, que puede ser un espacio agrario periurbano. Aunque todavía no es muy común en el Estado Español, en el resto de Europa y en Norteamérica es una práctica cada vez más extendida.

Finalmente, las experiencias de planificación quedarían definidas atendiendo a su condición de política pública adoptada por la administración con respecto a un área determinada. Aunque anteriormente se ha situado a la planificación como una de las responsables de la degradación actual de los espacios agrarios periurbanos, también se ha señalado la existencia de excepciones que demuestran la posibilidad de planificar estos espacios con cierto éxito, incluyendo también la participación social como un elemento importante tal y como se va a describir a continuación.

En muchos de los ejemplos descritos, una experiencia particular incluye características de las tres tipologías, ya que se trata de procesos en los que se superponen diferentes actores y escalas espaciales y temporales. En la selección realizada sobre la gran cantidad de experiencias existentes, se han tratado de considerar las que señalaban esta superposición y las que permitían explicar mejor cada una de las tipologías (incluyendo la diversidad existente dentro de éstas). Evidentemente, como cualquier estudio que aborda una temática global, es lógico que las condiciones de quien lo escribe (profesor en una universidad española) y del medio de publicación (revista española escrita en esta lengua) impliquen una atención especial a los casos del ámbito europeo (sobre todo mediterráneo) y de Latinoamérica.

\subsection{Experiencias ciudadanas}

\subsubsection{Movimientos ecologistas y culturales}

Se trata de grupos que defienden activamente el paisaje y el territorio, y que desde la década de los 70 del siglo pasado (algunos países se incorporan más tarde) forman redes muy activas tanto en la denuncia como en la propuesta (Martínez Alier, 1995; Nel·lo, 2006; Cruz Gallach, 2008; Observatorio Metropolitano, 2011). Atendiendo al crecimiento de la conciencia de lugar y a la importancia creciente de cuestiones como la sostenibilidad urbana 
y territorial, o la soberanía alimentaria, los espacios agrarios periurbanos constituyen cada vez más, lugares de atención prioritaria en las agendas reivindicativas de estos movimientos.

En el caso español muchos de estos grupos han estado vinculados de una forma $u$ otra con Ecologistas en Acción y sus federaciones, cuyas asociaciones destacan por haber trabajado activamente en la defensa de los espacios agrarios periurbanos. Aunque se podrían glosar decenas de experiencias, cabría destacar dos: la Plataforma Salvemos la Vega de Granada que aparte de paralizar numerosas agresiones (lamentablemente, no todas) a este espacio, ha conseguido que la administración regional esté elaborando actualmente un Plan Especial para el Parque Agrario de la Vega de Granada (Matarán Ruiz, Russo Cardozo, Martínez Parra, y Paniagua Guzmán, 2010), y la Plataforma Per l'Horta de Valencia que también ha sido capaz de forzar a la administración regional (en este caso dirigida por el otro partido mayoritario a nivel estatal) a elaborar el Plan de Acción Territorial de la Huerta. En ambos ejemplos, la administración todavía no ha terminado de aprobar los planes y tampoco está dando suficiente voz a estos colectivos más allá de jornadas puntuales de participación a pesar de la cantidad de propuestas y de actividades que han realizado para proteger y dinamizar los espacios agrarios periurbanos.

En el plano internacional, son numerosos los casos de movimientos ecologistas y culturales preocupados por los espacios agrarios periurbanos, de hecho, muchas de las iniciativas que se describen en los dos subapartados siguientes nacen de estos colectivos y de las experiencias ciudadanas que activan. Como ejemplos en el ámbito europeo se podrían destacar en Italia la asociación ecologista Legambiente y la cultural Arci, que han fomentado los parques agrarios participando además en su desarrollo (Fanfani, 2009), y en el Reino Unido, la asociación Common Ground y la red Sustain: The alliance for better food and farming, que junto a otras están promoviendo la agricultura urbana y periurbana (Verdaguer, 2010).

\subsubsection{Asociaciones vecinales, barriales, agrarias y campesinas}

También serán importantes las experiencias activadas por las asociaciones vecinales y barriales que faciliten el cuidado del territorio, de los espacios públicos y de la calidad de vida o que simplemente quieren alimentarse mejor (Bryld, 2003; Parker, 2005; Lamine, 2008; Sánchez Hernández, 2009).

Se cuentan por decenas los grupos que trabajan en esta tarea en el Estado Español (Morán, 2010), aunque cabe destacar algunas experiencias que plantean nuevas formas de ocupar los espacios urbanos ya sean públicos o privados, con una importancia creciente en las grandes capitales como por ejemplo las del Parque Miraflores (Carmona Gallego, 2004) y Parque Tamarguillo en Sevilla, en las que los grupos vecinales incluyen entre sus demandas la recuperación de las agriculturas periurbanas como forma de reapropiación activa del espacio público y como método para favorecer la recuperación de la memoria biocultural y la calidad alimentaria. También existen numerosos huertos urbanos creados por colectivos sociales en los espacios agrarios periurbanos, algunos de ellos ligados a Centros Sociales Ocupados como Canmasdeu en Barcelona; aunque en el área metropolitana de esta gran ciudad mediterránea (Domene y Saurí, 2007) la mayor superficie total corresponde a ciudadanía con la que a priori no se cuenta: personas jubiladas, gente que emigró del campo a la ciudad, y otros grupos, que han ocupado o, en menor medida, alquilado espacios periurbanos 
para practicar la agricultura como estrategia de ocio, de autonomía alimentaria e incluso en algunos casos, de complemento de rentas (sobre todo en momentos de crisis como el actual).

También es necesario hacer una mención especial a las asociaciones de personas que se dedican a la agricultura, las cuales en el Estado Español no han participado de forma generalizada en los espacios agrarios periurbanos (con algunas excepciones como la Uniò de Pagessos en la experiencia del Parque Agrario del Baix Llobregat que se describirá más adelante), aunque en otros países sí se han implicado como ocurre con las organizaciones agrarias francesas en el proyecto Terres en Villes (que también se va a describir a continuación), o la elaboración por parte de la Confederación Italiana de Agricultores de la «Carta para la agricultura periurbana», que está vinculada al trabajo de esta organización y otros colectivos para fomentar la protección y el desarrollo de los espacios agrarios periurbanos, llegando a participar en acciones concretas junto a estos otros colectivos ciudadanos para construir desde la base proyectos territoriales (ver más adelante las experiencias italianas en materia de planificación).

Sin embargo, a nivel internacional, al igual que en otras luchas sociales, destaca la efervescencia latinoamericana donde la fuerza de los movimientos barriales (Zibechi, 2008) y campesinos (Desmarais, 2008) en algunos casos se orienta a los espacios agrarios periurbanos, tanto para mejorar la calidad de vida de las maltratadas periferias de las grandes (y pequeñas) ciudades latinoamericanas como para buscar nuevas formas de autonomía laboral y soberanía alimentaria al igual que sucede en otros países periféricos tal y como se ha resaltado en la introducción. En ocasiones se vinculan a la tradición campesina de las comunidades originarias (Zibechi, 2006) aunque siempre están relacionadas con la respuesta a los efectos devastadores de las políticas neoliberales que se les imponen a través de los llamados ajustes estructurales (Davis, 2006) como en el caso argentino que se describe a continuación. Antes de seguir, cabe destacar el caso cubano ya que ha sido paradigmático por su capacidad de resistir al imperialismo a través del desarrollo estatal y también autónomo de las agriculturas urbanas y periurbanas (Altieri, et al, 1999; González Novo y Murphy, 2001; Cruz, 2002; Santandreu y Dubbleing, 2002), que en 1999 llegaron a producir de forma ecológica el 65\% del arroz, el 46\% de las verduras frescas y el 38\% de las frutas cubanas (Santos, 2010).

En Argentina la situación es muy diferente. En este país del Cono Sur, conviven experiencias cooperativas, de autoconsumo y de autoempleo algunas de ellas ayudadas y/o promovidas por el estado a través del Plan Pro-Huerta, que es un programa dirigido a la población en condición de pobreza, que enfrenta problemas de acceso a una alimentación saludable, promoviendo una dieta más diversificada y equilibrada mediante la autoproducción en pequeña escala de alimentos frescos por parte de sus destinatarios. También incluye asesoramiento para cultivos orgánicos de autoconsumo a nivel familiar, escolar, comunitario e institucional (Instituto Nacional de Tecnología Agropecuaria, 1991).

En el caso particular del Gran Buenos Aires (con 1500 establecimientos agrarios dentro de este plan) donde tras décadas de regresión, se está produciendo un crecimiento de las agriculturas gracias a que el $40 \%$ está en manos de bolivianos que en una situación de grave crisis económica y mediante un cierto sentido colectivo (organizados en asociaciones o en otras formas más o menos colectivas) están recuperando los modelos agrarios tradicionales que incluyen cauces diversos (formales e informales) de comercialización (Benencia, et al, 2009). Volviendo al apoyo institucional, en el partido de Pilar se ha creado un programa de buenas prácticas agropecuarias para estas personas (Barsky, 2005). 


\subsubsection{Grupos de producción, consumo y/o autoconsumo}

Por último, también existe un número creciente de grupos de producción y consumo de productos locales, donde incluso las personas más comprometidas también se encargan de partes de la producción. Siendo de carácter muy diverso, están proliferando en las ciudades de los países centrales relacionados con las incipientes redes de soberanía alimentaria (Calori, 2009; Binimelis, 2010), y vinculados principalmente a sus espacios agrarios periurbanos, aunque según su configuración también tienen relaciones con otros grupos o personas productoras de comarcas rurales algo más alejadas.

En el Estado Español destaca el caso de Cataluña con numerosas experiencias activas desde hace décadas (Binimelis, 2010, Vivas, 2010) como Germinal en Barcelona (http:// coopgerminal.coop). En el resto del país, algunos de los ejemplos comenzaron en los espacios agrarios periurbanos, por ejemplo el precursor BAH! (Bajo el Asfalto está la Huerta) en Madrid, Almocafre en Córdoba, o el más reciente grupo Hortigas en Granada aunque problemas con los precios del suelo y otros procesos urbanos hicieron que todos se desplazaran a zonas algo más alejadas; sin embargo otras experiencias que comenzaron vinculadas al mundo rural como La Ortiga en Sevilla que nació junto con la Cooperativa la Verde localizada en una zona rural (la Sierra de Cádiz) (Becerra Gómez, 2004) ahora mantienen relaciones también con personas que producen en el espacio agrario periurbano sevillano; por último, cabe destacar la experiencia recién iniciada de El Vergel de la Vega en Granada que ha nacido como un éxito más del movimiento en defensa de este territorio periurbano, en particular de las mujeres agricultoras y dinamizadoras que lo componen.

En el panorama internacional, destacan las TEIKEI fundadas por las mujeres de las grandes metrópolis de Japón que buscaban alimentos de calidad que no estaban disponibles en las tiendas y que serían las precursoras en los años 70 de este tipo de organizaciones (junto con otros grupos en Suiza y Alemania). En este caso, están estructuradas entorno a la Asociación Japonesa de Agricultura Ecológica que fue fundada en 1971, según datos de esta organización, en 2000 había 3000 personas asociadas, siendo un 20-25\% de ellas productoras (aunque no todas pertenezcan al espacio periurbano), y configurando hasta 1000 grupos según el momento (Parker, 2005).

También son importantes en el contexto europeo las francesas AMAP-Associations pour le Maintien de 1'Agriculture Paysanne (Asociaciones para la Conservación de la Agricultura Campesina). Tras su nacimiento en 2001 en el Área Metropolitana de Marsella han seguido un extraordinario proceso de desarrollo: actualmente, puede haber entre 70.000 y 140.000 personas vinculadas con las AMAP, y rondan el millar de asociaciones según la fuente (Lamine, 2008). Existe una gran demanda de agricultura de proximidad en las ciudades (Aubry, Kebir, y Pasquier, 2008), tanta que en muchos casos como en la Región de Îlle de France (Área Metropolitana de París) es difícil de cubrir existiendo largas listas de espera para algunas AMAP (Lamine, 2008). Por lo tanto, es evidente que estas organizaciones se están convirtiendo en un sujeto activo de la transformación de los espacios agrarios periurbanos a través de la puesta en valor y el mantenimiento de las agriculturas, su transformación (mejora económica, ambiental y/o social), o su reintroducción. También existen AMAP en Bélgica, bajo la denominación de Groupes d'Achats Solidaires de l'Agriculture Paysanne. 
En el ámbito anglosajón este tipo de organizaciones se denominan CSA (Community Supported Agriculture) y existen en EE.UU., Canadá, Australia e Inglaterra. Sus características y su nivel de desarrollo es similar al de otros países centrales (Feagan y Henderson, 2009), destacando los EE.UU., un país donde las iniciativas agroalimentarias alternativas tienen una larga trayectoria (Allen, FitzSimmons, Goodman, y Warner, 2003), y en el que existen unos 1500 grupos de CSA que en su mayor parte están vinculados con los espacios agrarios periurbanos (McFadden, 2004).

Por último cabe señalar a los Grupi di Acquisto Solidale (Grupos de compra solidaria) en Italia, destacando que éstos últimos están participando en los proyectos de parques agrarios que se describirán más adelante.

\subsection{Experiencias de pactos entre la administración y la ciudadanía}

El ejemplo más importante que se ha encontrado en el contexto español, son las redes de custodia del territorio, que se forman gracias a la colaboración entre administraciones, entidades de custodia del territorio (incluidas algunas de carácter ciudadano) y personas que poseen los terrenos sobre los que se produce el acuerdo (Cordón y Sánchez, 2010). Aunque no constituyen la mayoría de los casos, sí que existen algunos acuerdos en el contexto de los espacios agrarios periurbanos, ya que la conservación del patrimonio y los paisajes agrarios forman parte de los objetivos prioritarios de estas redes (Pietx y Basora, 2009), pudiendo destacarse aquí el Parc Agrari de Sabadell que lleva funcionando desde 2005 con la colaboración de estas redes de custodia del territorio.

A nivel internacional, la idea pactista se ha desarrollado de modo particular en Francia y Bélgica donde los pactos toman la forma de «Contrat de pais» $\mathrm{y}$ «Contrat de Rivière» (que han sido exportados con éxito para dar lugar a los «Contratti di fiume» italianos). En el caso francés también están vinculados a la formación de asociaciones (que implican a los tres sectores descritos) como las Terres en Ville que incluye a la rama agraria de los gobiernos regionales (APCA), a la Federación de Tierras y Sociedades Rurales (Société d'Aménagement Foncier et d'établissement Rural - FNSAFER) tipología francesa de las entidades de custodia del territorio, ya que entre sus cometidos se incluye el de facilitar el acceso a la propiedad de las fincas a los colectivos con proyectos de gestión territorial de interés público, y a la Asociación de Pastores (Bergerie Nationale) entre otras organizaciones agroganaderas; la red generada en los espacios periurbanos franceses ha permitido compartir experiencias, promocionar las agriculturas periurbanas y facilitar la aplicación de políticas públicas en este sentido (Bonnefoy, 2005)

También en Francia mediante estos pactos se generan instrumentos específicos de gobernanza (más o menos abierta a la participación de agentes diversos, según el caso) del territorio periurbano asociados a las políticas de gestión y ordenación territorial como las experiencias pactistas de los Programmes local de l'agriculture, los Protocole de partenariat, o las más conocidas Charte agricole que se refieren a un contrato que firman diferentes agentes con respecto a las actividades agrarias generalmente periurbanas y que se basa en la «libertad para los agricultores» que significa eliminar las restricciones urbanísticas del uso agrario (Poulot, 2008; Bonnefoy, Verdier, Chaucesse, Vargas, Marly, y Jullien, 2008). Otro ejemplo en esta línea lo constituyen los Programmes agro urbains (Poulot, 2008) de los que 
generalmente forman parte unos documentos de planificación y gestión territorial específicos para las agriculturas periurbanas denominados Projects Agri-Urbains (Vidal y Fleury, 2008; Duvernoy, et al, 2005) cuyo objetivo es mantener las explotaciones agrarias dado su valor paisajístico, patrimonial, ambiental y de espacio abierto metropolitano para usos productivos, educativos, de ocio, de integración social...

En los Estados Unidos se han desarrollado los llamados Collaborative community efforts to preserve farmaland y otras experiencias de custodia del territorio como las vinculadas al American Farmland Trust (www.farmland.org) que en algunos casos están vinculadas a los espacios agrarios periurbanos que se consideran muy amenazados por el modelo urbano norteamericano (Esseks, et al, 2009). En ocasiones se trata de iniciativas nacidas desde la base que consideran el papel central de las personas dedicadas a las agriculturas y que llegan a organizarse en torno a la creación de comités ciudadanos (Fanfani y Magnaghi, 2010).

\subsection{Experiencias de planificación}

\subsubsection{Los parques agrarios}

Se trata de espacios agrarios periurbanos que han sido calificados como tales por las administraciones competentes en materia urbana y territorial. Incluyen iniciativas de gestión y de dinamización de las agriculturas a las que se añaden otras propuestas de uso multifuncional del espacio (Fanfani y Magnaghi, 2010). A diferencia de algunas formas de proteger el territorio, el Parque Agrario no pretende momificar el territorio, si no dinamizarlo de forma ordenada.

La primera experiencia española en la materia es la del Parque Agrario del Baix Llobregat que existe desde 1998 en el área metropolitana de Barcelona, tiene 2.938 ha, de las cuales 1.969 el $67 \%$ son agrarias. Este parque es el resultado claro de la participación social de las personas que se dedicaban y se dedican a la agricultura en este espacio (Callau y Montasell, 2009). Así ha sido desde que a finales de los años setenta realizarán la primera campaña con el lema ;Salvem el Pla! hasta que se crea el consorcio del parque con la participación de las organizaciones agrarias (aunque no de otras asociaciones o personas interesadas) y gracias a la voluntad de un grupo de municipios y del gobierno provincial como en el caso milanés que se describe a continuación. La colaboración entre quienes se dedican a la agricultura en este espacio y quienes lo gestionan es constante, de tal manera que muchas de las política públicas desarrolladas tienen que ver con la mejora de las condiciones de cultivo y con el incremento de las posibilidades de comercialización de la producción agraria. Además, la reciente amenaza de desaparición de este espacio por la construcción de un megaproyecto de casinos y centros de congresos (denominado Eurovegas), ha sido respondida mediante la participación de numerosas personas y colectivos que agrupados en la Plataforma Aturem Eurovegas han sido un factor más para que se haya descartado esta propuesta en el área metropolitana barcelonesa.

A nivel internacional, la experiencia decana en esta materia es la del Parque Agrario del Sur de Milán (Parco Agricolo Sud Milano), nacido en 1990 gracias a la suma de la voluntad de numerosos municipios y de la provincia de Milán, ocupa unas 47.000 ha, de las cuales 39.916, el 85 \% están dedicadas a la agricultura. El profesor Andrea Calori (2010) alude como factor determinante a la participación ciudadana vinculada a un incremento en la con- 
ciencia de lugar (en una región innovadora y activa socialmente durante esos años) como rechazo a los procesos de degradación producidos por la expansión incontrolada y desordenada de la metrópoli milanesa. La idea siempre fue la de poner en valor tanto las agriculturas como el patrimonio ambiental y cultural de este espacio, contando por tanto con un apoyo creciente de las personas dedicadas a la agricultura. De hecho, en el artículo 14 de los estatutos del parque se acuerda la presencia de tres representantes agrarios en un Comité Técnico Agrícola que debe opinar sobre todo aquello que afecte a la actividad agrícola. Aunque no es un ente de gestión propiamente dicho, se asemeja mucho y facilita la participación constante de las personas dedicadas a la agricultura. Sin embargo, según el artículo 17, la participación del resto de la ciudadanía (sindicatos, asociaciones ecologistas, empresariales, culturales, juveniles...) queda resuelta con la publicación de los proyectos en curso y con la consulta, eso sí, orientada a la implicación de estas personas en dichos proyectos.

Según Andrea Calori (2010) en la actualidad el parque pasa por una situación de impás debida tanto a los cambios institucionales como a la fuerza de las presiones especulativas acrecentadas con la celebración de la Expo 2015 en Milán. Sin embargo, el fortalecimiento del parque está llegando desde la base gracias a la participación social que se está desarrollando en torno a la relación entre el campesinado más innovador y los cada vez más numerosos Gruppi di Aquisto Solidale (GAS) a los que se ha hecho referencia anteriormente. Además, tanto las energías ciudadanas que sustentan el parque como otros movimientos sociales milaneses junto con personas de la universidad están promoviendo que la Expo 2015, a priori dedicada a la sostenibilidad, sea de verdad un revulsivo para mejorar tanto el parque como la ciudad con otro tipo de iniciativas similares que cambien la tendencia insostenible ligada a la especulación inmobiliaria y a la expansión urbana que sigue la metrópoli milanesa.

La última experiencia que cabe destacar en este subapartado es la del Parque Agrario de Prato en Toscana (Italia), descrita con gran detalle en el libro editado por David Fanfani en 2009. Aunque este parque todavía no ha sido creado de forma efectiva por la administración, al nacer desde la base, las asociaciones y personas que lo promovieron y que consiguieron que el Ayuntamiento lo incluyera como espacio agrario en su Plan de Ordenación Urbana, se han asociado en un Forum por el Parque (Forum per il Parco) y, junto con la Universidad de Florencia, están activando proyectos que de hecho construyen el propio parque llegando hasta el punto de que la administración regional está actualmente desarrollando la idea con varios municipios. Se trata por tanto de un claro ejemplo en el que la participación social constituye un pilar fundamental para la sostenibilidad y para las políticas públicas en los espacios agrarios periurbanos.

\subsubsection{Los anillos verdes}

Se pueden considerar como la primera experiencia de planificación moderna que incluye a las agriculturas periurbanas, aunque su origen tiene que ver con la contención de la urbanización y con la mejora de los bordes urbanos, más que con la dinamización de los espacios agrarios periurbanos (Kühn, 2003; Amati, 2008)

El anillo verde de Barcelona constituye uno de los primeros experimentos serios de construir una estructura de este tipo en el contexto español. Aunque está basado principalmente en los espacios forestales que circundan la aglomeración barcelonesa, incluye entre sus 150.000 
ha, algunos espacios agrarios como el Parque Agrario del Baix Llobregat (Montseny, 1999) razón por la cual se ha considerado este anillo en el elenco de ejemplos.

A nivel internacional, aunque existen anillos verdes en muchos lugares, destacan por su antigüedad y por su importancia los británicos, que comprenden 13.050 .388 ha en 14 ciudades, incluido el de Londres que tiene 484.173 ha. (Verdaguer, 2010). El éxito de esta medida de planificación a la hora de contener el crecimiento urbano ha sido escaso, aunque desde el punto de vista de la gestión de estos espacios el 51\% de las autoridades locales británicas afirmaba en 2002 que los anillos verdes funcionan adecuadamente (Ravetz, 2002).

En el caso londinense, sólo un $57 \%$ (274.564 ha) del anillo verde tiene uso agrícola (Verdaguer, 2010). Y dentro de esta clasificación, nos encontramos con que un $51 \%$ (144.741 ha) del suelo agrícola está cubierto por las iniciativas de financiación pública para el fomento de las prácticas agroambientales, y 831 ha constituyen huertos urbanos (cuya función es complementada por la existencia de 8 Granjas Urbanas) (Garnett, 2001) . Un reciente informe avisa de importantes riesgos para la agricultura periurbana londinense, aunque existe una visión positiva dentro del mundo agrario a cerca de las posibilidades de la multifuncionalidad y de la participación social (Adas UK Ltd, 2005).

Con respecto a dicha participación, actualmente las autoridades y otros agentes sociales del territorio han implementado El Enfoque Arco Verde: Mejorando el entorno rural de Londres (The Green Arc Approach: Improving the countryside around London), que incluye entre otras cosas la aplicación de Protocolos de custodia del territorio (Environmental Stewardship) en colaboración con las personas propietarias de los terrenos, incluyendo las dedicadas a la agricultura.

\subsubsection{Los espacios naturales protegidos}

Se trata de una categoría en la que la planificación de los espacios agrarios periurbanos se aborda atendiendo a la protección de zonas (agrarias y no agrarias) en función de sus valores ambientales y culturales, tal y como se ha hecho históricamente en lugares menos poblados. La larga tradición de la protección de espacios naturales implica la existencia de una importante diversidad de modelos, aunque en los espacios periurbanos se podrían destacar los casos de Holanda y Francia, por su nivel de desarrollo en el ámbito de las agriculturas periurbanas.

En el caso español se han encontrado pocas referencias donde estas agriculturas fueran una parte fundamental y un argumento clave del espacio protegido en cuestión. Entre ellas destacan el Parque Natural de La Albufera de Valencia donde los arrozales son un componente esencial, constituyendo el mantenimiento de esta actividad y la reducción de los impactos que produce, dos de los principales desafíos a los que se enfrenta la administración del parque. Además, la presión de la ciudadanía, por ejemplo a través de la Plataforma Per L’Horta de Valencia (señalada anteriormente) supone una importante vigilancia de los impactos ambientales y de las amenazas existentes sobre este espacio periurbano. Igualmente, los proyectos de dinamización de las agriculturas activados por esta plataforma (agricultura ecológica, canales cortos de comercialización, jornadas de estudio...) implican la consolidación de esta actividad tanto en el ámbito del parque como en ámbitos cercanos, generando 
con ello dinámicas favorables para el conjunto del territorio. Por último, cabe destacar que el Plan de Acción Territorial para La Huerta de Valencia (en fase de elaboración) desarrollado como respuesta a la presión ciudadana, incluye dentro de su espacio de actuación el Parque Natural de la Albufera de Valencia.

En el ámbito internacional cabe destacar el Corazón Verde holandes (Groene Hart) espacio abierto central con unos $1500 \mathrm{~km}^{2}$ de los cuales al menos 1.050 (un 75\%) son agrarios (Kühn, 2003) que existe dentro de una de las zonas más densamente urbanizadas de Europa, el Randstad, que es una red urbana en forma de anillo donde se incluyen las cuatro mayores ciudades holandesas (Amsterdam, La Haya, Rotterdam y Utrecht).

En un principio la protección de los suelos agrarios que cubren la mayoría de este espacio estaba ligada a la protección de la producción agrícola, incluso proponiendo la autosuficiencia alimentaria. En la actualidad, con la intensificación y la dedicación de las tierras para pastos (Kühn, 2003), la protección se plantea desde la preservación de los valores ambientales y del paisaje. De este modo, se busca la multifuncionalidad del espacio agrario, con una gestión que incorpore los servicios verdes (relacionados con la naturaleza) y azules (relacionados con la gestión del agua) y que se vincule a la demanda de espacios verdes y de ocio de las ciudades (Van Rij, 2009).

La conciencia de lugar en Holanda está muy desarrollada (Van Dijk, Aarts y De Wit, 2009), y el movimiento en defensa de este territorio en particular desarrolló el manifiesto Groene Randstad Vs Deltametropolis (Ranstad Verde frente a la planificación de una gran metrópolis urbana en esta región) que está influyendo en la dinamización multifuncional de las agriculturas y en el rechazo de los cambios en la planificación que permitan una mayor urbanización del Groene Hart.

Además, en la actualidad se reconoce que las personas dedicadas a la agricultura y la ganadería han sido protagonistas esenciales en la configuración del paisaje y que sin ellas desaparecería, por lo tanto se están desarrollando acuerdos con estos grupos (en ocasiones organizados en poderosas cooperativas) que incluyen ayudas económicas, apoyo técnico, bancos de suelo, evaluación agraria, y el programa Farming for nature (Agricultura para la naturaleza) que incluye 100.000 ha donde se apoya el papel ambiental de los espacios agrarios en todo el país (Callau, 2009).

En el caso de la planificación francesa, sobre la base de la experiencia pactista descrita anteriormente, aparecen dos instrumentos muy interesantes para los espacios agrarios periurbanos: el primero serán las Zonas Agrícolas Protegidas (Zones agricoles protégées - ZAP) que en algunas ocasiones han sido consideradas en áreas metropolitanas (Bonnefoy, et al, 2008); el segundo, más específico, serán los Perímetros de protección y puesta en valor de los espacios agrícolas y naturales periurbanos (Périmètres de protection et de mise en valeur des espaces agricoles et naturels périurbains - PAEN) que implican por ejemplo la posibilidad de que la entidad de custodia del territorio local (SAFER o Sociedad de Gestión Territorial y de los Establecimientos Rurales) ejerza el derecho de tanteo sobre estos territorios (Simón Rojo, 2010).

Por último, cabría destacar los Parques Naturales Regionales (Parcs Naturals Regional) desarrollados en los espacios periurbanos a partir de la primera experiencia de 1985 (HauteVallée de Chevreuse en la Región de París, Île-de-France), que toman medidas similares a los parques agrarios, incluyendo una gestión participada con los agentes del territorio (Vidal y 
Fleury, 2008), dado que la tradición de estos parques está muy vinculada a las agriculturas en zonas rurales donde éstas tenían un alto valor y en algunos casos problemas para mantenerse.

\subsubsection{Otros casos}

El carácter particular de la planificación anglosajona implica la existencia de otras formas de abordar los espacios agrarios periurbanos, en las que en muchas ocasiones es necesaria la voluntad manifiesta de las personas dedicadas a la agricultura.

Destacan los Distritos Agrarios (Agrarian Districts) cuya participación implica dicha voluntariedad en los EE.UU. aunque en Canadá es de carácter obligatorio una vez que la administración ha decidido calificar como Distrito Agrario una zona (Paül, 2009). Tienen por objetivo la ordenación del espacio agrario (incluyendo ayudas) para el mantenimiento de la actividad y en algunos casos de los paisajes (Franzen, 2006).

En EE.UU. se utiliza también el instrumento del Purchase Development Rights (pago por no urbanizar) y del Transfer Development Rights (pago por urbanizar en otro lugar) que es de carácter voluntario, lo cual implica necesariamente la participación de quienes poseen la tierra, aunque al depender del mercado no siempre funciona de forma adecuada (Nelson, Fowler, y Dorfman, 2001).

No se han encontrado referencias a la existencia de una vinculación directa entre otras experiencias de participación social en los espacios periurbanos norteamericanos como las Community Supported Agriculure y estos instrumentos de planificación, aunque no se puede descartar la verosimilitud de la hipótesis de que exista una superposición entre los espacios agrarios periurbanos atendidos por la administración y los dinamizados por la ciudadanía.

\section{CONCLUSIONES}

La sostenibilidad debe llegar a las aglomeraciones urbanas donde vive la mayoría de la población del mundo (Davis, 2006). En este sentido, es esencial una vuelta a la centralidad del territorio que permita una reconstrucción de los límites de las ciudades favorecida por nuevas relaciones con sus bioregiones respectivas en las que ya se están activando procesos de territorialización por parte de algunas de las experiencias ciudadanas descritas en este artículo e incluso por parte de poderes públicos preocupados por la calidad de vida.

Este proceso será más traumático y más lento en las ciudades que hayan destruido gran parte de su patrimonio, consumiendo por ejemplo el suelo agrario que las rodeaba y alimentaba tradicionalmente, y que ha sido el lugar preferente de la expansión metropolitana desenfrenada de las últimas décadas. Sin embargo, todavía es importante en muchos de estos espacios la identidad de las centralidades preexistentes lo que, junto con el desarrollo de las experiencias ciudadanas ligadas al contexto barrial o local, podría facilitar el desarrollo de otras maneras de hacer para la recuperación de estos núcleos y de los espacios agrarios que los rodean (Magnaghi, 2011). Además, en este punto podría ser esencial el proyecto de recuperación del espacio público y de redefinición de los límites urbanos que algunos movimientos y autoridades locales están desarrollando, en algunos casos, vinculados a las cuestiones agrarias.

Para abordar con éxito todas estas cuestiones y conseguir la sostenibilidad de los espacios agrarios periurbanos debe quedar claro que la participación social no es sólo una exigencia 
democrática, si no una urgencia y que tanto los agentes fuertes como sobre todo los débiles (por su predominio en el cuidado de estos espacios), deben jugar su papel en esta construcción de escenarios de futuro sostenibles, como de hecho ya está ocurriendo en muchas de las ciudades del mundo donde la participación social está demandando, manteniendo o activando múltiples experiencias.

En lo que respecta a las experiencias de planificación, tanto las que están consolidadas como las que no, es necesario construir procesos de participación social constantes, que podrían estar ligados a experiencias pactistas entre la administración y los diferentes agentes del territorio que ya han tenido éxito en contextos muy diversos.

En cualquier caso, la estrategia de apoyar la sostenibilidad y, por tanto, las resistencias a los procesos neoliberales, pasa por el incremento de la participación social, implicando a la mayor cantidad de personas posibles, y para este fin se ha demostrado muy útil la centralidad de la alimentación en los análisis y las propuestas comunitarias y económicas (Desmarais, 2008) tanto en el ámbito urbano y periurbano, como en la creación de fronteras espaciales al capitalismo en universos rurales que defienden su autonomía frente al modelo de desarrollo imperante (Fernández Durán, 2011).

Por último, aunque el crecimiento de la conciencia de lugar genera experiencias ciudadanas alentadoras que están transformando la reivindicación en proyecto territorial, la participación social, tal y como se explica en este artículo, no es la panacea ya que se encuentra con numerosos problemas, como muchas otras cuestiones complejas de nuestro tiempo, debidos entre otras razones, a la debilidad, la pluralidad, y la fragmentación de los agentes implicados (Fanfani, 2009).

\section{BIBLIOGRAFÍA}

ADAS UK Ltd. (2004): Farming in London's Green Belt. Londres. London Development Agency.

ALLEN, P., FITZSIMMONS, M., GOODMAN, M., y WANER, K. (2003): «Shifting plates in the agrifood landscape: the tectonics of alternative agrifood initiatives in California». Journal of Rural Studies, vol. 19, pp. 61-75.

ALTIERI, M.I., COMPANIONI, N., CAÑIZARES, K., MURPHY, C., ROSSET, P., BOURQUE, M., y NICHOLLS, C.I. (1999): «The greening of the «barrios»: Urban agriculture for food security in Cuba». Agriculture and Human Values, Vol 16. pp. 131-140. ALTIERI, M., y NICHOLS, C.I. (2000): Agroecología: Teoría y práctica para una agricultura sustentable. Méjico. Programa de las Naciones Unidas para el Medio Ambiente, Serie Textos Básicos para la Formación Ambiental.

AMATI, M. (2008): Green Belts: A Twentieth-century Planning Experiment. En Amati, M. Urban Green Belts in the Twenty-first Century. Urban Planning and Environment. Ashgate Publishers.

AUBRY, C., KEBIR, L., y PASQUIER, C. (2008): «Short supply chains in periurban zones: a way to maintain rurality near the city? some examples taken in the Ille-de-France Region». En DEWAELHEYNS, V., y GULINCK, H., (Eds). Rurality near the city. Proceedings of the international conference and workshops held in Leuven, Belgium, on February 7-8th 2008. 
BALDESCHI, P., y POLI,D. (2008): «Agricoltura e paesaggio». Rivista Contesti 2008. Vol 1,

BARSKY, A., (2005): «El periurbano productivo, un espacio en constante transformación. Introducción al estado del debate, con referencias al caso de Buenos Aires». Scripta Nova, Revista Electrónica de Geografía y Ciencias Sociales. [En línea]. Barcelona: Universidad de Barcelona, 1 de agosto de 2005, vol. IX, n 194. <http://www.ub.edu/geocrit/ sn/sn-194-36.htm>.

BENENCIA, R., QUARANTA, G., y SOUZA CASADINHO, J. (2009): Cinturón Hortícola de la Ciudad de Buenos Aires. Cambios sociales y productivos. Buenos Aires. Ed. Ciccus.

BERNAL CARRETERO, F. (2010): Finanzas Alternativas, Éticas y Solidarias. El caso de Andalucía. Sevilla. Atrapasueños.

BIEHL, J., Y BOOKCHIN, M. (2009): Las políticas de la ecología social: Municipalismo libertario. Bilbao. Ed. Virus.

BINIMELIS, R. (2010): Circuitos cortos de comercialización para una agricultura de proximidad. Fundación Agroterritori. Seminario Agroterritorial. Castelldefels (Barcelona), 8 y 9 de Septiembre de 2010.

BONNEFOY, S., VERDIER, P., CHAUCESSE, J.N., VARGAS, L., MARLY, J., y JULLIEN, B. (2008.i): Prender en compte l'agriculture et ses espaces dans les SCOT. Certu. Terres en Villes- Éditions du Certu.

BONNEFOY, S. ( 2005): «French periurban agriculture: The Terres en Villes network, and the experiences of the participant cities». En Proceedings of the technical meeting of FEDENATUR, «Periurban agriculture: problems, opportunities and challenges», Bruselas 3 de Junio de 2005.

BRYLD, E. (2003): «Potentials, problems, and policy implications for urban agriculture in developing countries». Agriculture and Human Values, 2003, vol 20, pp. 79-86.

CALORI, A. (2009): «Del parco agricolo alla regione milanese: Empowerment degli attori per la riconquista della sovranità alimentare». En FANFANI, D. Pianificare tra città $e$ campagna. pp. 91-114. Florencia. Firenze University Press.

CALLAU, S. (2009): «Els spais agraris periurbans a Europa. El cas d’Holanda». En ALFRANCA, O., y PUJOLÀ, M. Agricultura Periurbana. Barcelona. Ed. Universidad Politécnica de Cataluña.

CALlAU, S., y MONTASELL, J. (2009): «Il Parco Agricolo del Baix Llobregat di Barcelona. Uno strumento di conservazione, gestione e sviluppo di uno spazio agricolo periurbano». En FANFANI, D. «Pianificare tra città e campagna». pp. 75-90. Florencia Firenze University Press.

CARMONA GALLEGO, J., (2004): «Del dicho al hecho... ¿hemos andado ese trecho?: Veinte años de lucha ciudadana por el Parque Miraflores». En ENCINA, J., ROSA, M., y ÁVILA, M. A., Democracias participativas e intervención social comunitaria desde Andalucía. Sevilla. Ed. Atrapasueños.

COMITÉ ECONÓMICO Y SOCIAL EUROPEO. (2004): Dictamen sobre la agricultura periurbana. Bruselas. CESE.

CORDÓN, M. y SÁNCHEZ, A. (2010): Informe del 2o Inventario de Iniciativas de Custodia del Territorio del Estado español. Madrid. Plataforma de Custodia del Territorio de la Fundación Biodiversidad. 
CRUZ GALLACH, E. (2008): «Conflictos territoriales y movilizaciones ciudadanas: algunas reflexiones sobre las formas de gobernanza territorial actuales». Boletín de la AGE $\mathrm{n}^{\circ} 48$. pp 375-387.

CRUZ, M.C. (2002): «Agricultura urbana: ordenamiento territorial en la Ciudad de La Habana.» En Anuario de Ecología y Sociedad, 2002. №2. pp. 113-126.

DAVIS, M. (2006): Planeta de ciudades miseria. Madrid. Ed. Foca.

DELIND, L.B., (2002): «Place, work, and civic agriculture: Common fields for cultivation». Agriculture and Human Values 2002. vol. 19, pp. 217-224.

DESMARAIS, A. A. (2008): «The power of peasants: Reflections on the meanings of La Vía Campesina». Journal or Rural Studies 2008, vol. 24, pp. 138-149.

DEWAELHEYNS, V., y GULINCK, H. (2008): Rurality near the city. Proceedings of the international conference and workshops held in Leuven, Belgium, on February 7-8th, 2008.

DOMENE, E. y SAURÍ, D. (2007): «Urbanization and class-produced natures: Vegetable gardens in the Barcelona Metropolitan Region». Geoforum 2007, n⿳ 38, pp. 287-298.

DONADIEU, P. (2008): «Paesaggio, urbanistica e agricoltura: dalle logiche economiche agricole alle logiche paesaggistiche urbane». Rivista Contesti 2008, vol. 1, pp. 39-50. Florencia (Italia).

DUVERNOY, I., JARRIGE, F., MOUSTIER, Y., y SERRANO, J. (2005): «Une agriculture multifonc-

tionnelle dans le projet urbain: Quelle reconnaissance, quelle gouvernance». Les Ca-hiers de la Multifonctionnalité 2005, nº, pp. 87-104.

ESSEKS, D., OBERHOLTZER, L., CLANCY, K., y LAPPING, M. (2009): Sustaining Agriculture in Urbanizing Counties: Insights from 15 Coordinated Case Studies. Lincoln. University of Nebraska-Lincoln.

EUROPEAN ENVIRONMENT AGENCY, (2006): Land accounts for Europe 1990-2000. Bruselas. Office for Official Publications of the European Communities.

FAO. (2007): «Profitability and sustainability of urban and peri-urban agriculture». Agricultural managemente, marketins and finance Occasional Paper 19.

FANFANI, D. (2009): Pianificare tra città e campagna. Florencia. Firenze University Press.

FANFANI, D. y MAGNAGHI, A. (2010): «Il parco agricolo, un nuovo strumento per la pianificazione del territorio aperto». En MAGNAGHI, A. y FANFANI. D. Patto città campagna: Un progetto di bioregione urbana per la Toscana centrale. Florencia. Ed. Alinea.

FARIÑA TOJO, J. (2011): «El plan de urbanismo ante los límites del crecimiento. Necesidad de nuevos instrumentos para organizar la ciudad del siglo XXI». En LÓPEZ, F. y MATARÁN, A. La Tierra no es muda: Diálogos entre el desarrollo y el postdesarrollo. Granada. Editorial Universidad de Granada.

FEAGAN, R., y HENDERSON, A. (2009): «Devon Acres CSA: local struggles in a global food system». Agriculture and Human Values 2009. vol. 26. pp 203-217.

FERNÁNDEZ DURÁN, R. (2006): El Tsunami urbanizador español y mundial. Bilbao. Ed. Virus.

FERNÁNDEZ DURÁN, R. (2011): El Antropoceno: La expansión del capitalismo global choca con la biosfera. Bilbao. Ed. Virus. 
FORNS PUCH, N. (2008): «Horts urbans i horticultura terapèutica». En NOQUÉ, J., PUIGBERT, L., y BRETCHA, G. Paisatge i salut. Olot. Serie: 'Reflexions' Observatorio del Paisaje de Cataluña.

FRANZEN, E. (2006): Agricultural Zoning as a Farmland Protection Tool in Georgia. Atlanta. University of Georgia.

FUNES MONZOTE, F., (2010): «Transición hacia la agricultura sostenible en Cuba». En GASCÓN. E. y MONTAGUT, X. (Coords.). Cambio de rumbo en las políticas agrarias latinoamericanas. Barcelona. Ed. ICARIA.

GARNIER, J.P. (2006): Contra los territorios del poder. Bilbao. Ed. Virus.

GARNETT, T. (2001): «Urban agriculture in London: Rethinking our food economy». En BAKKER, N., DUBBELING, M., GUENDEL, S., SABEL KOSCHELLA, U., y DE ZEEUW, H. (eds.) Growing Cities, Growing Food, Urban Agriculture on the Policy Agenda. Feldafing. DSE.

GIARRACA, N., y TEUBAL, M. (2009): La tierra es nuestra, tuya y de aquel... Las disputas por el territorio en América Latina. Buenos Aires. Ed. Antropofagia.

GONZÁLEZ DE MOLINA, M. (2009): El desarrollo de la agricultura ecológica en Andalucía (2004-2007). Crónica de una experiencia agroecológica. Barcelona. Ed. Icaria.

GONZÁLEZ NOVO, M. y MURPHY, C. (2001): «Urban agriculture in the city of Havana: a popular response to a crisis». En BAKKER, N., DUBBELING, M., GUENDEL, S., SABEL KOSCHELLA, U., y DE ZEEUW, H. (eds.) Growing Cities, Growing Food, Urban Agriculture on the Policy Agenda. Feldafing. DSE.

INSTITUTO NACIONAL DE TECNOLOGÍA AGROPECUARIA. (1991): Plan ProHuerta. Buenos Aires. INTA.

INWOOD, S.M., SHARP, J.S., MOORE, R.H., y STINNER, D.H. (2009): «Restaurants, chefs and local foods: insights drawn from application of a diffusion of innovation framework». Agriculture and Human Values 2009, vol. 26. pp. 177-191.

JAROSZ, L. (2008): «The city in the country: Growing alternative food networks in Metropolitan areas». Journal of Rural Studies 2008, vol. 24, pp 231-244.

KÜHN, M. (2003): «Greenbelt and Green Heart: separating and integrating landscapes in European city regions». Landscape and Urban Planning 2003, vol. 64, pp. 19-27

LAMINE, C. (2008): Les AMAP: un nouveau pacte entre producteurs et consumateurs?. Gap. Yves Michel.

LATOUCHE, S. (2007): La otra África: Autogestión y apaño frente al mercado global. Barcelona. Oozebap.

LATOUCHE, S. (2009): Decrecimiento y posdesarrollo: El pensamiento creativo contra la economía del absurdo. Barcelona. Ed. El Viejo Topo.

LUGINBÜHL, Y. (2008): «Paisatge i benestar individual i social». En NOQUÉ, J., PUIGBERT, L., y BRETCHA, G. Paisatge i salut. Olot. Serie: 'Reflexions' Observatorio del Paisaje de Cataluña.

MAGNAGHI, A. (2011): El Proyecto Local. Barcelona. Ed. Universidad Politécnica de Cataluña.

MARTÍNEZ ALIER, J. (1995): De la economía ecológica al ecologismo popular. Barcelona. Ed. Icaria. 
MARTÍNEZ ALIER, J. (1998): La economía ecológica como ecología humana. Madrid. Ed. Fundación César Manrique.

MATARÁN RUIZ, A., RUSSO CARDOZO, F., MARTÍNEZ PARRA, E., y PANIAGUA GUZMÁN, J.A. (2010): «New Metropolitan Mainstreams: Granada». En INURA Conference 2010. Zurich (Suiza), 27 de Junio-3 de Julio de 2010.

MCFADDEN, S. (2004): The history of community supported agriculture part II: CSA's world of possibilities. Washington. Newfarm.

MICHELINI, J. J., (2010): «Gobernanza territorial, iniciativas locales y desarrollo urbano: los casos de Getafe y Álcazar de San Juán». Boletín de la AGE. n54, pp. 175-201.

MONTSENY, A. (1999): «Anella verda, a green belt around Barcelona Metropolitan Area». Planeco Project Newsletter $\mathrm{n}^{\circ} 3$.

MORÁN, N. (2010): «Semillas de resistencia en el asfalto estéril». Periódico DIAGONAL n ${ }^{\circ}$ 124, Sábado 24 de Abril de 2010. Madrid.

NAREDO, J. M., (2008): Economía, Poder y Megaproyectos. Lanzarote. Ed. Fundación César Manrique.

NAREDO, J. M., (2009): Luces en el Laberinto: Autobiografía intelectual. Madrid. Ed. Los Libros de la Catarata.

NEL·LO, O. (2006): «Aquí no! Los conflictos territoriales en Cataluña. Orígenes, dinámica y alternativas». En TARROJA, J. y CAMAGNI, R. (Coords), Una Nueva Cultura del Territorio. Criterios sociales y ambientales en las políticas y el gobierno del territorio. Barcelona. Ed. Diputación de Barcelona.

NELSON, N., FOWLER, L., y DORFMAN, J. (2001): Protecting Farmland in Developing Communities. Atlanta. University of Georgia.

OBSERVATORIO METROPOLITANO. (2011): La crisis que viene: Algunas notas para afrontar esta década. Madrid. Ed. Traficantes de Sueños.

PARKER, G. (2005): «Sustainable food? Teikkei, Co-operatives and food citizenship in Japan and the UK». Working papers in Real Estate \& Planning 11/05, April 2005.

PÉREZ SERRANO, J., CASTAÑO MADROÑAL, A., y MANJAVACAS RUIZ, J.M. (2009): Democracia, proyecto urbano y participación ciudadana. Los presupuestos participativos de Sevilla y otras experiencias andaluzas relevantes. Sevilla. Ed. Centro de Estudios Andaluces.

PIETX, J., y BASORA, X. (2009): «La custòdia del territori: un nou instrument de concertació basat en la implicació ciutadana per a conservar el paisatge». En NOQUÉ, J., PUIGBERT, L., y BRETCHA, G. Ordenació i gestió del paisatge a Europa. Olot. Observatori del Paisatge de Catalunya. (Plecs de Paisatge; Eines; 2).

PAÜL, V. (2009). «Bones pràctiques d'ordenaciò dels spais agraris periurbans». En ALFRANCA, O., y PUJOLÀ, M. Agricultura Periurbana. Barcelona. Ed. Universidad Politécnica de Cataluña.

POULOT, M. (2006): «Les programmes agri-urbains en Île-de-France: de la «fabrique» de territoires périurbains». Colloque La dynamique des territoires en milieu périurbain et le patrimoine naturel et culturel 26-28 abril 2006 Université de Montréal.

RAVETZ, J. (2002): Sustainable Development of the Countryside Around Towns. Manchester. N.Yorks, Countryside Agency Publications. 
RENTING, H., MARSDEN, T. K., y BANKS, J. (2003): «Understanding alternative food networks: exploring the role of short food supply chains in rural development». Environment and Planning A 2003, vol. 35, nº 3, pp. 393-411.

RIECHMANN, J. (2009): La habitación de Pascal: Ensayos para fundamentar éticas de suficiencia y políticas de autocontención. Madrid. Ed. Los Libros de la Catarata.

RIECHMANN, J. y FERNÁNDEZ BUEY, F. (1994): Redes que dan libertad: Introducción a los nuevos movimientos sociales. Barcelona. Ed. Paidós.

RODA, R. (2009): «Los territorios peiriurbanos: un hecho territorial creciente en Europa». En ALFRANCA, O., y PUJOLÀ, M. Agricultura Periurbana. Barcelona. Ed. Universidad Politécnica de Cataluña.

RUBINO, A. (2008); «Nuove forme e modalità di integrazione tra agricoltura e città». Rivista Contesti 2008, vol 1, pp. 85-89.

SALDIVAR-TANAKA, L., y KRASNY, M.E. (2004): «Culturing community development, neighborhood open space, and civic agriculture: The case of Latino community gardens in New York City». Agriculture and Human Values 2004, vol. 21, pp. 399-412.

SÁNCHEZ HERNÁNDEZ, J.L. (2009). «Redes alimentarias alternativas: Concepto, tipología y adecuación a la realidad española». Boletín de la AGE nº49, pp. 185-207.

SANTANDREU, A. y DUBBLEING, M. (2002): «Proceso participativo y constructivo de diagnóstico para la agricultura urbana». Anuario de Ecología y Sociedad, 2002 №2. pp.127-138.

SANTOS, M. (2010): «Decrecimiento y migraciones». En TAIBO, C. Decrecimientos: sobre lo que hay que cambiar en la vida cotidiana. Madrid. Ed. Los Libros de la Catarata.

SERREAU, C. (2010): Solutions locales pour un désordre global. Saint-Etienne. Ed. Actes Sud.

SIMÓN ROJO, M. (2010): «La protección del suelo agrícola en la Comunidad de Aglomeración de Montpellier (Francia)» En VÁZQUEZ, M., y VERDAGUER, C. El espacio agrícola entre el campo y la ciudad. Madrid. Ed. Boletin $\mathrm{CF}+\mathrm{S}$. Universidad Politécnica de Madrid.

SMIT, J., RATTA, A., y NASR, J., (1996): Urban Agriculture: Food, Jobs and Sustainable Cities. Nueva York. UNDP, Habitat II Series.

TOLEDO, V.M., y BARRERA-BASSOLS, N., (2008): La memoria biocultural: La importancia ecológica de las sabidurías tradicionales. Barcelona. Ed. Icaria.

UNITED NATIONS ENVIRONMENTAL PROGRAM. (2005): One Planet, Many People: Atlas of Our Changing Environment. Paris. Le Monde Diplomatique.

VALENZUELA, L.M. PÉREZ CAMPAÑA, R., AGUILERA BENAVENTE, F. and MATARÁN, A. (2007): «Assessment of the incidence of landscape ecology in urban and spatial planning in Southern Europe: revision of Spanish and Italian case studies». International Association for Landscape Ecology (IALE), Congress 2007. Wageningen (Netherlands), 8-12 ${ }^{\text {th }}$ July 2007. Vol II. pp. 1016-1017.

VALENZUELA MONTES, L.M., PÉREZ CAMPAÑA, R., y MATARÁN RUIZ, A. (2009): «Ecoestructura y multifuncionalidad del paisaje agrourbano». Revista Ciudades 12. 2009, vol. 12. pp. 67-95.

VAN DIJK, T., AARTS, N., y DE WIT, A. (2009): «Spaces of engagement for open space advocacy: a grounded theory on local opposition in the Netherlands». En VAN DER 
VALK, A., y VAN DIJK, T. Regional PLanning for Open Space. Londres. The RTPI Library Series - Routledge.

VAN RIJ, E. The viability of cross-subsidy strategies: a Netherlands case study. En VAN DER VALK, A., y VAN DIJK, T. Regional PLanning for Open Space. Londres. The RTPI Library Series Routledge. 2009.

VAN DER PLOEG, J. D., (2010): Nuevos campesinos: Campesinos e imperios alimentarios. Barcelona. Ed. Icaria.

VERDAGUER, C. (2010): «Planificación del desarrollo y preservación de los usos agrícolas en el Gran Londres (Gran Bretaña)». En VÁZQUEZ, M., y VERDAGUER, C. El espacio agrícola entre el campo y la ciudad. Madrid. Ed. Boletin $\mathrm{CF}+\mathrm{S}$. Universidad Politécnica de Madrid.

VIDAL, R., y FLEURY, A. (2008): «Agriculture in urban planning in Île-de-France». DEWAELHEYNS, V., y GULINCK, H. Rurality near the city. Proceedings of the international conference and workshops held in Leuven, Belgium, on February 7-8th, 2008

VIVAS, E. (2010): «Consumo agroecológico, una opción política». Viento Sur n ${ }^{\circ} 108$, Febrero de 2010, pp. 54-62.

VIVAS, E. (2011): «Del anticapitalismo y el ecologismo como alternativa política». En LÓPEZ, F. y MATARÁN, A. La Tierra no es muda: Diálogos entre el desarrollo y el postdesarrollo. Granada. Editorial Universidad de Granada.

ZIBECHI, R. (2006): Dispersar el poder. Buenos Aires. Ed. Tinta Limón.

ZIBECHI, R. (2008): Territorios en resistencia. Cartografía política de las periferias urbanas latinoamericanas. Buenos Aires. Ed. Lavaca. 
